\title{
Motor Memory Consolidation in Sleep Shapes More Effective Neuronal Representations
}

\author{
Stefan Fischer, ${ }^{1}$ Matthias F. Nitschke, ${ }^{2}$ Uwe H. Melchert, ${ }^{3}$ Christian Erdmann, ${ }^{2}$ and Jan Born ${ }^{1}$ \\ Departments of ${ }^{1}$ Neuroendocrinology, ${ }^{2}$ Neurology, and ${ }^{3}$ Neuroradiology, University of Lübeck, D-23538 Lübeck, Germany
}

Learning a motor skill involves a latent process of consolidation that develops after training to enhance the skill in the absence of any practice and crucially depends on sleep. Here, we show that this latent consolidation during sleep changes the brain representation of the motor skill by reducing overall the neocortical contributions to the representation. Functional magnetic resonance brain imaging was performed during initial training and $48 \mathrm{~h}$ later, at retesting, on a sequential finger movement task with training followed by either a night of regular sleep or sleep deprivation. An additional night of sleep for all subjects served to rule out unspecific effects of sleep loss at retrieval testing. Posttraining sleep, but not sleep deprivation, led to improved motor skill performance at retrieval. This sleep-dependent improvement was linked to greatly reduced brain activation in prefrontal, premotor, and primary motor cortical areas, along with a stronger involvement of left parietal cortical regions. Our findings indicate that storing a motor skill during sleep reorganizes its brain representation toward enhanced efficacy.

Key words: memory; motor system; sleep; skill learning; fMRI; human

\section{Introduction}

Learning a new motor skill is considered to establish an internal model representing the required spatial-temporal motor output to the different muscles (Wolpert et al., 1995; Shadmehr and Holcomb, 1997). Depending on the specific task demands, the internal representation thereby corresponds to an activation of selected neuronal networks, including motor and nonmotor brain areas, while performing the skill. Acquiring a skill essentially relies on at least two distinct processes, fast learning and a slow latent process of consolidation (Karni and Sagi, 1993; McGaugh, 2000; Ungerleider et al., 2002; Walker, 2004). Whereas fast learning refers to the improvement in performance occurring during ongoing practice within a training session, a slow and latent process of consolidation evolves after practice has finished, allowing the long-lasting storage of the skill. In the absence of any intermittent practice, the slow latent consolidation does not only result in a stabilization but in an enhanced performance at later retesting (Karni and Sagi, 1993; Karni et al., 1998; Korman et al., 2003). Importantly, this "off-line" improvement in motor skill learning has been demonstrated to crucially depend on sleep. Specifically, the skill at later retesting is substantially enhanced only if initial training is followed by sleep on the subsequent night, indicating that this first posttraining sleep period plays an essential role for the long-lasting storage of the skill (Karni et al.,

Received May 2, 2005; revised 0ct. 17, 2005; accepted 0ct. 17, 2005.

This work was supported by grants from the Deutsche Forschungsgemeinschaft and the VolkswagenStiftung to M.F.N. and J.B. We thank C. Büchel for critical reading of a previous version of this manuscript and A. Otterbein and B. Rasch for their skillful technical assistance.

Correspondence should be addressed to Jan Born, Department of Neuroendocrinology, University of Lübeck, Ratzeburger Allee 160, Haus 23a, D-23538 Lübeck, Germany. E-mail: born@kfg.uni-luebeck.de.

DOI:10.1523/JNEUROSCI.1743-05.2005

Copyright $\odot 2005$ Society for Neuroscience $\quad$ 0270-6474/05/2511248-08\$15.00/0
1998; Gais et al., 2000; Stickgold et al., 2000; Fischer et al., 2002; Walker et al., 2002).

There is consistent evidence that fast learning within a training session, as well as the gradual acquisition of a skill across repeated training sessions, is associated with significant changes in skill representation in various brain regions relevant to performance (Seitz et al., 1990; Jenkins et al., 1994; Elbert et al., 1995; Karni et al., 1995; Jueptner et al., 1997b; Graybiel, 1998; Toni et al., 1998; Hund-Georgiadis and von Cramon, 1999). These findings point to plastic mechanisms and a reorganization of the involved networks underlying the learning process (Elbert et al., 1995; Karni et al., 1995; Shadmehr and Holcomb, 1997). However, in most previous studies investigating effects of repeated training sessions, contributions of within-session fast learning were intermingled with those of slow latent consolidation, although there are exceptions. Thus, Penhune and Doyon (2002) identified dynamic changes in the brain representation of a motor skill that occurred in the absence of additional practice 4 weeks after initial training at retrieval testing. However, attempts to isolate changes in motor skill representation that occur specifically in conjunction with sleep-dependent consolidation processes have been made so far in only two studies, one using a complex visuomotor task (Maquet et al., 2003) and the other using a paced finger-sequence tapping task (Walker et al., 2005). Both of them revealed postlearning sleep-dependent changes in memory representations. Here, we aimed to further dissect the pure effect of consolidating a basal motor skill in memory during sleep on the brain representation of this skill.

\section{Materials and Methods}

Participants and experimental protocol. Subjects were 20 young healthy volunteers (18-28 years; mean $\pm \mathrm{SD}, 21.4 \pm 4.1$ years; 12 females). One-half of them participated in the main experiments with retrieval 
testing taking place $48 \mathrm{~h}$ after initial training (at 10:00 P.M.) (see Fig. $1 B$ ). The other one-half was examined in supplementary experiments with task performance taking place at 10:00 P.M. (initial training) and then again at 7:00 A.M. (retesting) (i.e., immediately before and after a nocturnal interval in which subjects slept or remained awake). Because, in the main experiments ( $48 \mathrm{~h}$ retention interval), two subjects were to be excluded from the analyses, one because of excessive movement artifacts in the functional magnetic resonance brain imaging (fMRI) data and the other because of a daytime nap, the resulting number of subjects was $n=$ 8 in the main experiments and $n=10$ in the supplementary experiments. According to within-subject designs in both experiments, subjects were tested twice, once in the posttraining sleep and once in the posttraining wake condition. To allow for testing of the same subject in both conditions, different motor sequences (A; B) (see Fig. 1 A) were trained on each occasion. The subject's experimental conditions were separated by at least 4 weeks. The order of retention conditions and of motor sequences was balanced across subjects. Sleep during the first night after training was recorded polysomnographically. To control for subjective mood and feelings of activation and tiredness at training and retrieval, testing subjects were tested with an adjective checklist after the scanning sessions (Janke and Debus, 1978).

Subjects were used to sleeping $7-8 \mathrm{~h}$ per night and had no disruption of their regular sleep/wake rhythm during the 6 weeks before the experiments. All were right-handed, as assessed by the Edinburgh Handedness Inventory (Oldfield, 1971). None had ever practiced playing a musical instrument nor was trained as a typist. Subjects were not allowed to ingest any caffeine or alcohol on the days of the experiment. They were also instructed to get up at 7:00 A.M. and not to take any naps during these days. Before participating in the study, subjects gave written informed consent. The experiments were approved by the Ethics Committee of the University of Lübeck.

Finger-sequence tapping task. The finger-sequence tapping task used in all experiments was a finger-to-thumb opposition task. It required subjects to tap (against the thumb), with their nondominant hand (left), a five-element finger sequence as rapidly and as accurately as possible. Before the initial training session in the MRI scanner, all subjects were made familiar with the task by practicing the respective finger sequence for 5 min under the supervision of the experimenter. Initial training and retrieval testing were scheduled according to a block design with $30 \mathrm{~s}$ of rest (eight intervals) alternating with $30 \mathrm{~s}$ of task performance (seven intervals). To allow imaging at the individual's optimum performance, the first $6 \mathrm{~s}$ in each block were considered an adaptation period and were not included in the analysis. Two different motor sequences were used to allow testing of the same subject in both the sleep and wake conditions (see Fig. 1A). The order of conditions and of finger sequences was balanced across subjects. Experimental conditions were at least 1 week apart. Motor skill performance was measured in terms of performance rate (correctly completed sequences per $30 \mathrm{~s}$ ) and error rate (mean number of errors per $30 \mathrm{~s}$ ). Performance improvements were determined by the difference in performance rate and accuracy between initial training and retrieval testing, and were also transformed to percentages, with the individual performance at training set to $100 \%$.

Sleep recordings and actimetry. Sleep was recorded polysomnographically during the first night after training and analyzed according to standard criteria (Rechtschaffen and Kales, 1968). To further assure that subjects did not sleep during daytime and rested during the second night, physical activity of the subjects was monitored from the beginning of the training session until retrieval testing using actimetry (Actiwatch; Cambridge Technology, Cambridge, UK).

$f M R I$ data acquisition and analyses. Imaging was performed on a $1.5 \mathrm{~T}$ Magnetom Symphony scanner (Siemens, Erlangen, Germany). A total of $74 \mathrm{~T} 2{ }^{*}$-weighted functional volume images were acquired in each 7.5 min session with a gradient echoplanar sequence using an axial slice orientation along the anterior and posterior commissural line (repetition time, $6 \mathrm{~s}$; echo time, $81 \mathrm{~ms} ; 128 \times 128 \times 38$ voxels; $2 \times 2 \times 4 \mathrm{~mm}^{3}$ voxel size).

Imaging data were analyzed using statistical parametric mapping (SPM2; Wellcome Department of Cognitive Neurology, London, UK). Functional volumes from each session were corrected for head motion and spatially normalized to an EPI template conforming to the Montreal Neurological Institute (MNI) space. Images were then spatially smoothed using a Gaussian kernel of $8 \mathrm{~mm}$ full-width at half-maximum and high-pass filtered $(1 / 132 \mathrm{~Hz})$ to remove participant-specific drifts in the signal. Mean activity evoked by the condition effects ("motor task performance" and "rest") were used as regressors in a multiple regression analysis. To reduce motion-induced artifacts, movement parameters as yielded from the realignment procedure were added as covariates of no interest. In each subject, the effects of interest were computed by linear contrasts, generating statistical parametric maps of $t$ values. The resulting individual contrast images were then entered into a second-level analysis, corresponding to a random effects model, to be able to generalize the observed effects to the underlying population. Analyses were performed using one-sample $t$ tests. Differential effects of posttraining sleep versus sleep deprivation were assessed by the training/retrieval by sleep/sleep deprivation interaction using within-subject ANOVAs. Using inclusive masking, two separate ANOVAs were calculated for voxels showing increased and decreased brain activation in the main effect of training/ retrieval (i.e., training $>$ retrieval and training $<$ retrieval) $(p<0.001$, respectively). This analysis permits us to distinguish specifically greater increases from lesser decreases in brain activation (and vice versa) during retrieval testing with reference to initial training. Then, we compared patterns of brain activation elicited during training and retrieval separately for the posttraining sleep and wake conditions. Brain regions in which activation significantly increased in both the sleep and the wake condition were identified by calculating a conjunction analysis. For this purpose, we first created a mask only containing voxels in which activation significantly increased from initial training to retrieval testing in both the posttraining sleep and wake conditions $(p<0.001$, uncorrected). In a subsequent overall ANOVA, the main effect of training/ retrieval was restricted to these voxels using inclusive masking. To demonstrate similarities between the sleep-dependent decreases in brain activation across both the 48 and $8 \mathrm{~h}$ retention intervals, we performed a conjunction analysis using the training $>$ retrieval contrast from the posttraining sleep condition in the main study as an inclusive mask $(p<$ 0.001 ). It should be noted that our significant conjunctions do not mean that all the contrasts were individually significant (i.e., a conjunction of significance). It simply means that the contrasts were consistently high and jointly significant. This is equivalent to inferring that one or more effects were present (Friston et al., 2005). Furthermore, we show that the sleep-dependent changes in brain activity identified in our analyses pertain to areas that have been reported previously to be activated during performance of sequential finger movement tasks. On the basis of these a priori locations, we corrected respective $p$ values for small volume on a 10 $\mathrm{mm}$ sphere around the corresponding published coordinates $\left(p_{\mathrm{SVC}}\right): 41$, 32, 14 for the right prefrontal cortex (BA 46) (Jenkins et al., 1994; Peigneux et al., 2000); 35, 44, 9 for the right prefrontal cortex (BA 10) (Jenkins et al., 1994; Jueptner et al., 1997a; Grafton et al., 2002); - 14, 36, 19 for the left prefrontal cortex (BA 9) (Grafton et al., 2002); 14, 32, 40 for the right anterior cingulate cortex (BA 32) (Grafton et al., 2002); 29, 7, 50 for the lateral premotor cortex (BA 6) (Jenkins et al., 1994; Peigneux et al., 2000; Penhune and Doyon, 2002); 35, - 19, 66 for the right primary motor cortex (BA 4) (Toni et al., 1998; Peigneux et al., 2000; Walker et al., 2005); -55, 6, 22 for the left primary motor cortex (BA 4) (Grafton et al., 2002; Dechent et al., 2004); 42, -21, 29 for the right primary somatosensory cortex (BA 2) (Peigneux et al., 2000; Grafton et al., 2002); -38, -12, 30 for the left primary somatosensory cortex (BA 2) (Peigneux et al., 2000); $-27,-47,55$ for the left superior parietal cortex (BA 7) (Jenkins et al., 1994; Jueptner et al., 1997b; Toni et al., 1998; Maquet et al., 2000); 13, 15, 7 for the right caudate nucleus (Jueptner et al., 1997a; Toni et al., 1998; Peigneux et al., 2000); -20, 9, 3 for the left putamen (Jueptner et al., 1997b; Peigneux et al., 2000); $-21,-39,-22$ for the left cerebellum (Toni et al., 1998; Maquet et al., 2000). Only clusters of $>30$ contiguous voxels are reported.

\section{Results}

Sleep and task performance

Polysomnographic recordings confirmed a regular sleep pattern in the first night succeeding the initial training on the sleep con- 
dition. In this night, subjects, on average, slept $396.88 \pm 11.34 \mathrm{~min}$ (mean \pm SEM). They spent $39.5 \pm 6.73 \mathrm{~min}(9.93 \pm$ $1.89 \%)$ in stage 1 sleep, $209.75 \pm 6.16 \mathrm{~min}$ $(52.99 \pm 2.24 \%)$ in stage 2 sleep, $84.5 \pm$ $9.96 \min (21.16 \pm 2.01 \%)$ in slow-wave sleep, and $63.63 \pm 9.36 \mathrm{~min}(15.93 \pm$ $2.08 \%$ ) in rapid eye movement (REM) sleep.

Finger sequence tapping at initial learning before the $48 \mathrm{~h}$ retention interval was very similar in the sleep and wake conditions with regard to both performance rate $(p>0.8)$ (Fig. $1 C)$ and accuracy $(p>$ $0.3)$. Although performance rates (i.e., the number of correctly completed sequences per $30 \mathrm{~s}$ ) improved from $20.02 \pm 0.45$ at initial training to $23.11 \pm 0.60$ (percentage of gain, $15.56 \pm 2.52)$ across retention intervals of sleep $\left(F_{(1,7)}=41.31 ; p<0.001\right)$, there was no similar change across the retention interval of sleep deprivation, with the performance rate averaging $20.41 \pm$ 1.49 at initial learning and $21.45 \pm 1.30$ $(5.86 \pm 2.84 \%)$ at retrieval testing $\left(F_{(1,7)}\right.$ $=3.51 ; p>0.1)$ (Fig. 1C). The increase in performance rate selectively across retention intervals of sleep was confirmed by a significant training/retrieval by sleep/sleep deprivation interaction $\left(F_{(1,7)}=6.01 ; p<\right.$ $0.05)$. The gain in performance rate after posttraining sleep was on average somewhat smaller than in a previous study using the same task (Fischer et al., 2002), which we attribute to the behaviorally restricted conditions in the MRI scanner. In parallel with performance rate, accuracy, as indicated by the number of errors per $30 \mathrm{~s}$, slightly improved from $2.91 \pm 0.27$ to $2.64 \pm 0.17(-2.39 \pm 12.30 \%)$ when subjects slept the first night after training. However, accuracy even deteriorated when subjects stayed awake during this first night, averaging $2.57 \pm 0.34$ before and $3.04 \pm 0.19(30.71 \pm 14.56 \%)$ after the retention interval $\left(F_{(1,7)}=5.86 ; p<0.05\right.$ for training/retrieval by sleep/sleep deprivation interaction). Because all subjects had slept the night before retrieval testing, effects of tiredness cannot explain the inferior retrieval performance after posttraining sleep deprivation. Accordingly, subjective ratings on an adjective checklist performed immediately after the scans confirmed closely comparable levels of "concentration," "activation," and "fatigue" on both conditions ( $p>0.9$, for all comparisons). Additional analyses (using Pearson's correlation) on data of the posttraining sleep condition did not reveal any significant relationship between time spent in the different sleep stages and performance gains.

\section{fMRI results}

Initial training of the finger motor skill was linked to a specific pattern of brain activation typical for the performance on this task (Karni et al., 1995, 1998; Hund-Georgiadis and von Cramon, 1999) (Table 1, Fig. 2A). Neocortical clusters of activation during task performance compared with rest covered the right and left
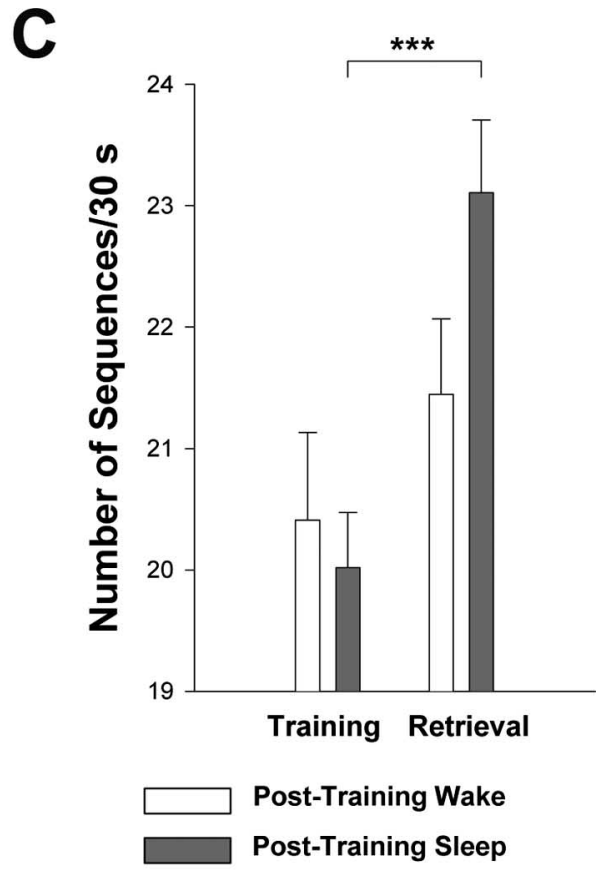

Night Day Night Day

\begin{tabular}{|c|c|}
\hline lake & $22-23 \mathrm{~h}$ \\
\hline eep & $22-23 \mathrm{~h}$ \\
\hline
\end{tabular}

Figure 1. Sequential finger-tapping task protocol of the main experiments and behavioral results. $\boldsymbol{A}$, Subjects had to oppose the fingers of the nondominant left hand to the thumb in two different finger sequences on the posttraining wake and sleep the main experiments, subjects $(n=8)$ performed the finger sequence task at 10:00 P.M. before (training) and after a $48 \mathrm{~h}$ (retrieval, black fields). During the first posttraining night, subjects either slept (gray areas) regularly or stayed closely comparable in both the sleep (gray bars) and the wake conditions (black bars). Whereas performance rate distinctly improved in the posttraining sleep condition, there was no significant change when subjects stayed awake the night after training. *** $p<0.001$. Error bars indicate SE.

cingulate cortices, the lateral premotor cortex, and the primary motor and somatosensory cortices. The right prefrontal cortex, right superior temporal cortex, left supplementary motor cortex, and left superior parietal cortex were also significantly more activated during initial training, compared with rest. Subcortical structures that were more activated during task performance than rest were the right and left caudate and cerebellum and the right putamen and ventral thalamus. Because the task was performed by our right-handed subjects with the left nondominant hand, activation in both hemispheres was nearly identical in most of the relevant motor and nonmotor areas (Kim et al., 1993; Hund-Georgiadis and von Cramon, 1999).

Effects of sleep-dependent latent consolidation on skill representations were assessed on the basis of the changes in brain activation at retrieval testing, with reference to initial learning (as indicated by the training/retrieval by sleep/sleep deprivation interaction). Most pronounced effects of posttraining sleep were revealed for areas with decreasing blood oxygen level-dependent (BOLD) signal from training to retrieval testing. Posttraining sleep, compared with sleep deprivation, significantly decreased retrieval-related brain activation in the right lateral premotor 


\begin{tabular}{|c|c|c|}
\hline Brain region & Coordinates of peak activation & $Z$ \\
\hline \multicolumn{3}{|l|}{ Prefrontal cortex } \\
\hline RBA 9 & $40,40,32$ & 2.84 \\
\hline \multicolumn{3}{|l|}{ Cingulate cortex } \\
\hline R BA 24 & $8,-6,30$ & 3.40 \\
\hline LBA 24 & $-14,-2,32$ & 3.44 \\
\hline \multicolumn{3}{|l|}{ Supplementary motor cortex } \\
\hline LBA 6 & $-14,-6,54$ & 4.22 \\
\hline \multicolumn{3}{|l|}{ Lateral premotor cortex } \\
\hline RBA 6 & $60,8,36$ & 3.67 \\
\hline LBA 6 & $-54,4,38$ & 3.06 \\
\hline \multicolumn{3}{|l|}{ Primary motor cortex } \\
\hline RBA 4 & $34,-20,40$ & 4.06 \\
\hline \multirow[t]{2}{*}{ LBA 4} & $-50,-18,36$ & 4.07 \\
\hline & $-30,-10,64$ & 3.86 \\
\hline \multicolumn{3}{|l|}{ Primary somatosensory cortex } \\
\hline RBA 2 & $40,-38,62$ & 4.87 \\
\hline LBA 2 & $-46,-24,22$ & 4.73 \\
\hline \multicolumn{3}{|l|}{ Temporal cortex } \\
\hline R BA 22 (Superior temporal cortex) & $48,0,2$ & 5.01 \\
\hline \multicolumn{3}{|l|}{ Parietal cortex } \\
\hline L BA 7 (Superior parietal cortex) & $-28,-56,66$ & 3.48 \\
\hline \multicolumn{3}{|l|}{ Subcortical activations } \\
\hline $\mathrm{R}$ caudate nucleus & $26,-36,6$ & 3.53 \\
\hline $\mathrm{R}$ caudate nucleus & $20,-8,22$ & 4.03 \\
\hline L caudate nucleus & $-16,18,18$ & 3.78 \\
\hline R putamen & $30,18,6$ & 3.27 \\
\hline R thalamus va & $2,-4,4$ & 2.57 \\
\hline R thalamus $\mathrm{dm} / \mathrm{p}$ & $2,-24,14$ & 3.34 \\
\hline R cerebellum & $28,-50,-30$ & 4.85 \\
\hline L cerebellum & $-6,-64,-50$ & 4.94 \\
\hline
\end{tabular}

All results are significant at $p<0.005$, uncorrected. R, Right hemisphere; L, left hemisphere; BA, Brodmann area; va, ventroanterior; $\mathrm{dm} / \mathrm{p}$, dorsomedial $/$ posterior.

cortex $\left(\mathrm{BA} 6 ; p_{\mathrm{SVC}}<0.05\right)$ and tended to decrease it in the right inferior primary somatosensory cortex (BA 2; $p<0.005$, uncorrected) (Table 2, Fig. $2 B$ ). In contrast, there was virtually no brain region yielding a larger decrease in brain response from training to retrieval testing in the posttraining wake than the posttraining sleep condition (Table 2, Fig. 2C). That only sleep after training induced a significant decrease in brain activation was confirmed by separate analyses of the sleep and the sleep deprivation conditions. This analysis revealed most prominent sleep-dependent decreases in activation in the left medial frontal lobe (BA 9) and the right lateral premotor cortex (BA $6 ; p_{\mathrm{SVC}}<0.05$, respectively). In addition, brain activation decreased in the right inferior frontal lobe (BA 46), the anterior cingulate cortex (BA 32), the left primary motor cortex (M1) (BA 4), and in an area in the right and left inferior primary somatosensory cortices (BA 2) (Table 2, Fig. 3). Although these latter effects reached an uncorrected significance level of $p<0.005$, they did not do so for small-volume corrected thresholds. When subjects were sleep deprived the night after training, significant decreases in the BOLD signal again were completely absent, even at a more liberal threshold $(p<0.01)$ (Table 2).

Some of the brain areas increasing in activation from initial training to retrieval testing were also found to be sensitive to the effect of posttraining sleep. Parameter estimates in the left superior parietal lobe (BA 7) showed significantly greater increases in activation at retrieval testing (with reference to training) when subjects had slept during the night after training than when they were sleep deprived $\left(p_{\mathrm{SvC}}<0.05\right)$. These analyses also revealed retrieval-related increases in brain activation that did not survive correction for multiple comparisons; posttraining sleep compared with sleep deprivation led to increases in BOLD response in the right caudate and the left anterior lobes of the cerebellum (Table 3, Fig. $2 D)$, whereas, vice versa, posttraining sleep deprivation selectively increased responses in the right middle frontal lobe (BA 10) and the left putamen (Table 3, Fig. 2E). Conjunction analyses identified the hand area in the right $\mathrm{M} 1$ to be the only region in which the BOLD contrast significantly increased from learning to retrieval testing in both the posttraining sleep and sleep-deprivation conditions $\left(p_{\text {SvC }}<0.05\right)($ Table 3, Fig. $2 F)$.

\section{Immediate retesting after sleep and sleep deprivation}

To assure that the changes in skill representation during latent consolidation indeed occur during sleep on the first posttraining night, in supplementary experiments, subjects were retested already in the morning after just one night of sleep and sleep deprivation. As expected, the nocturnal vigil here led to increased tiredness at retrieval testing. Despite this confound, the effects of interest of the main experiments were confirmed by these data. Performance rate across the sleep retention interval improved from $20.56 \pm 1.73$ (at training) to $22.30 \pm 1.94$ (at retrieval; percentage of gain, $8.46 \pm 3.36$ ), and error rate from $3.07 \pm 0.30$ to $2.17 \pm 0.23(-20.43 \pm 12.61 \%)$. In the wake condition, no improvement occurred. Here, the average performance rate was $20.41 \pm 2.26$ at training and $19.39 \pm 2.05$ at retesting $(-4.18 \pm$ $4.05 \%)$. The error rate averaged $2.93 \pm 0.23$ at training and $3.19 \pm 0.28$ at retesting $\left(17.40 \pm 16.21 \% ; F_{(1,9)}=6.93\right.$ and $F_{(1,9)}=6.00 ; p<0.05$ for training/retrieval by sleep/sleep deprivation interaction for performance rate and accuracy, respectively).

fMRI scans indicated that the selectively improved sequence tapping at retesting after posttraining sleep was again accompanied mainly by a decreased activation in cortical areas including relevant frontal motor sites. Compared with the wake condition, scans during retesting on the posttraining sleep condition revealed reduced activation in several frontal cortical regions including the right lateral premotor cortex (peak activation at 34, 18,$36 ; t=3.20$ ) and the supplementary motor cortex (peak activation at $6,18,68 ; t=3.60$ ) (Table 4 , Fig. $4 A$ ). In addition, a distinct decrease in brain activation was found in bilateral parietal areas and in the cerebellum. Notably, there were again no regions in which brain activation significantly decreased more in the posttraining wake as compared with the posttraining sleep condition (Table 4, Fig. 4B).

\section{The influence of tapping rate on brain activation}

To explore whether sleep-dependent changes in brain activation reflect increased speed of tapping at retrieval testing in this condition rather than acquired skill, in a supplementary analysis, we examined the influence of different finger tapping rates on patterns of brain activation independent of overnight learning. In this analysis, only data from initial training (of the main and supplementary experiments) were included, where subjects on both occasions showed comparable tapping speeds $(p>0.7)$, and were divided into two groups depending on whether subjects were high (mean performance rate, $23.53 \pm 1.12$ ) or low performers (mean performance rate, $17.20 \pm 0.29$ ). We then identified brain regions that were more (or less) activated in sessions with high compared with low performance rates. Although high performers had significantly faster tapping rates than low performers, they did not differ with respect to brain activation in any of the motor areas of interest, even at a very liberal threshold ( $p<$ 0.05 , uncorrected). Most importantly, however, there was also no single brain site in which activation decreased with increasing 

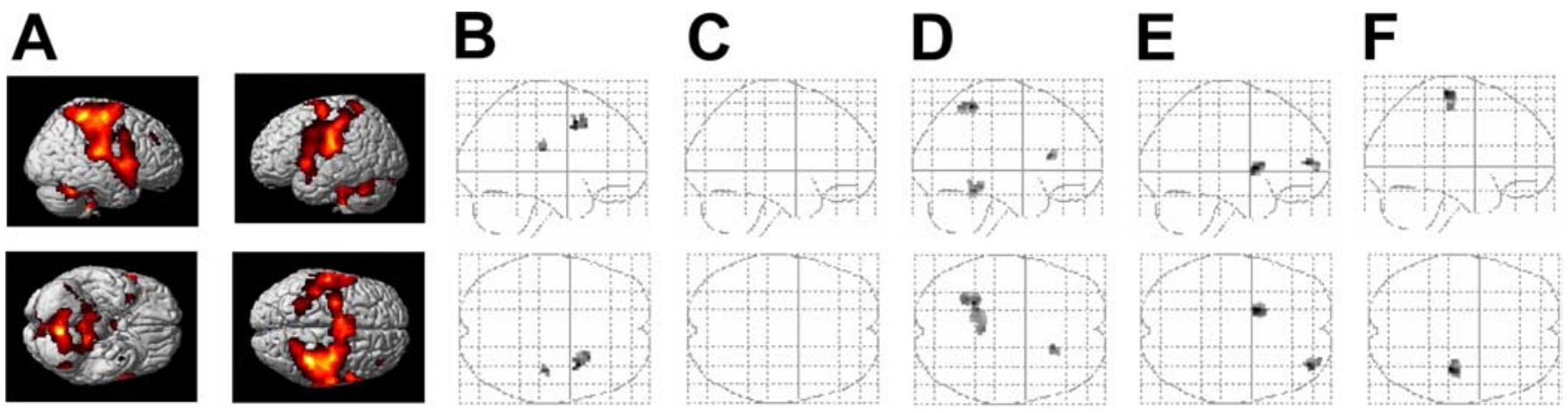

Figure 2. Statistical parametric maps (SPMs) of different contrasts in the main experiments ( $48 \mathrm{~h}$ retention interval; $n=8$ ). $\boldsymbol{A}$, Brain regions that were more activated during initial training of the finger motor task compared with rest. $\boldsymbol{B}$, Brain regions in which the decrease in retrieval-related activation with reference to training was larger in the posttraining sleep than in the posttraining wake condition and, vice versa, in which this decrease was larger in the posttraining wake than in the posttraining sleep condition $(\boldsymbol{C})$ (which never happened). $\boldsymbol{D}$, Brain regions in which the increase in retrieval-related activation across the retention interval was larger in the posttraining sleep than posttraining wake condition and, vice versa, in which this increase was larger in the posttraining wake than posttraining sleep condition $(\boldsymbol{E})$. $\boldsymbol{F}$, Brain regions of increased activation during retrieval testing with reference to initial training, regardless of whether subjects slept or stayed awake during the first night after training. SPMs are thresholded at $p<0.005$ (uncorrected) and displayed as sagittal and horizontal projections superimposed on the MNI standard brain and as glass brain views.

Table 2. Coordinates of local maxima for brain regions in which activation during finger motor performance decreased from new learning before to retrieval testing after a $48 \mathrm{~h}$ retention interval $(n=8)$

\begin{tabular}{|c|c|c|c|}
\hline Brain region & Coordinates of peak activation & Z & $p_{\text {SVC }}$ \\
\hline \multirow{2}{*}{\multicolumn{4}{|c|}{$\begin{array}{l}\text { Areas in which activation decreased more in the posttraining sleep than wake condition } \\
\text { Lateral premotor cortex }\end{array}$}} \\
\hline & & & \\
\hline RBA 6 & $34,12,44$ & 3.39 & 0.045 \\
\hline \multicolumn{4}{|c|}{ Primary somatosensory cortex } \\
\hline RBA 2 & $38,-22,20$ & 2.92 & 0.195 \\
\hline \multicolumn{4}{|c|}{$\begin{array}{l}\text { Areas in which activation decreased more in the posttraining wake than sleep condition } \\
\text { No suprathreshold clusters }\end{array}$} \\
\hline \multicolumn{4}{|c|}{ Areas in which activation selectively decreased in the posttraining sleep condition } \\
\hline \multicolumn{4}{|c|}{ Prefrontal cortex } \\
\hline RBA 46 & $44,24,12$ & 2.30 & 0.142 \\
\hline LBA 9 & $-10,36,24$ & 3.70 & 0.045 \\
\hline \multicolumn{4}{|c|}{ Anterior cingulate cortex } \\
\hline RBA 32 & $12,34,44$ & 3.28 & 0.189 \\
\hline \multicolumn{4}{|c|}{ Lateral premotor cortex } \\
\hline RBA 6 & $34,12,42$ & 4.76 & 0.002 \\
\hline \multicolumn{4}{|c|}{ Primary motor cortex } \\
\hline LBA 4 & $-62,-2,20$ & 2.86 & 0.160 \\
\hline \multicolumn{4}{|c|}{ Primary somatosensory cortex } \\
\hline RBA 2 & $40,-24,22$ & 3.36 & 0.092 \\
\hline LBA 2 & $-44,-12,32$ & 2.54 & 0.327 \\
\hline \multicolumn{4}{|c|}{$\begin{array}{l}\text { Areas in which activation selectively decreased in the posttraining wake condition } \\
\text { No suprathreshold clusters }\end{array}$} \\
\hline
\end{tabular}

All results are significant at $p<0.005$, uncorrected. $\mathrm{R}$, Right hemisphere; L, left hemisphere; BA, Brodmann area.

tapping rate. Thus, faster tapping speed, per se, cannot explain the pattern of change in brain activation observed at retrieval on the posttraining sleep condition.

\section{Discussion}

The most robust finding here is that sleep in the night after training induces pronounced decreases in the BOLD signal during retrieval compared with initial learning in various relevant neocortical areas. In contrast, when subjects had been awake on this night, there was no brain region at all in which activation was decreased at retesting. This pattern was equally revealed in the main study and in supplementary experiments, where retesting immediately followed sleep and sleep deprivation. The supplementary experiments, thus, ensure the importance of sleep on the first posttraining night to the enhancement in skill and associated changes in the cortical representation (Gais et al., 2000; Stickgold et al., 2000; Fischer et al., 2002), although, because of the additional effects of sleep deprivation, activation patterns from these supplementary experiments have to be interpreted with caution. Specifically, task-related patterns of brain activation immediately after sleep deprivation have been shown to reflect compensatory changes caused by fatigue and attentional deficits (Drummond et al., 2000). Consistent with our previous observations (Fischer et al., 2002), motor sequence performance after a $48 \mathrm{~h}$ retention interval was significantly superior in the posttraining sleep than posttraining wake condition. This finding strongly suggests that sleep improves skilled motor performance only within a certain time frame after training [i.e., that sleep is critical to the timing of the (behavioral) expression of the delayed gains in motor learning]. Previous findings indicating similar gains in skill $24 \mathrm{~h}$ posttraining, independent of whether subjects had been trained in the morning or in the evening before nocturnal sleep, suggest that the effects of training are maintained in some latent form during the waking state for at least $12 \mathrm{~h}$ (Korman et al. 2003). Indeed, similar findings were reported by others (Walker et al. 2003). The presence or absence of sleep, thus, appears to determine the time point in which latent processes of learning become effective. Initial training and retrieval testing taking place in our main experiments at the same time of day, and retention intervals comprising the same phases of the $24 \mathrm{~h}$ cycle, in addition, rule out that effects of circadian rhythm confounded memory performance and associated brain activation. Moreover, the $48 \mathrm{~h}$ delay in retrieval testing excluded confounding influences of tiredness at retesting, particularly in the wake condition, because all subjects had an additional night of sleep before retesting.

The prevalence of brain areas with decreasing activation at retrieval testing after sleep indicates that a central function of the latent consolidation process refers to an extinction of network activity that has become irrelevant for optimal performance. Specifically, the diminished contribution of right premotor and prefrontal areas at retesting in the posttraining sleep condition points to a decrease in the need for consciously regulating and self-monitoring the ongoing finger movements (Jenkins et al., 1994; Jueptner et al., 1997a, 1997b; Gehring and Knight, 2000). The right lateral premotor cortex in combination with prefrontal areas (here, particularly left BA 9) is involved in the planning, preparation, and selection of voluntary finger movements (Jueptner et al., 1997a; Hoshi and Tanji, 2000). It appears to be the site at which an internal model of the movement pattern is 


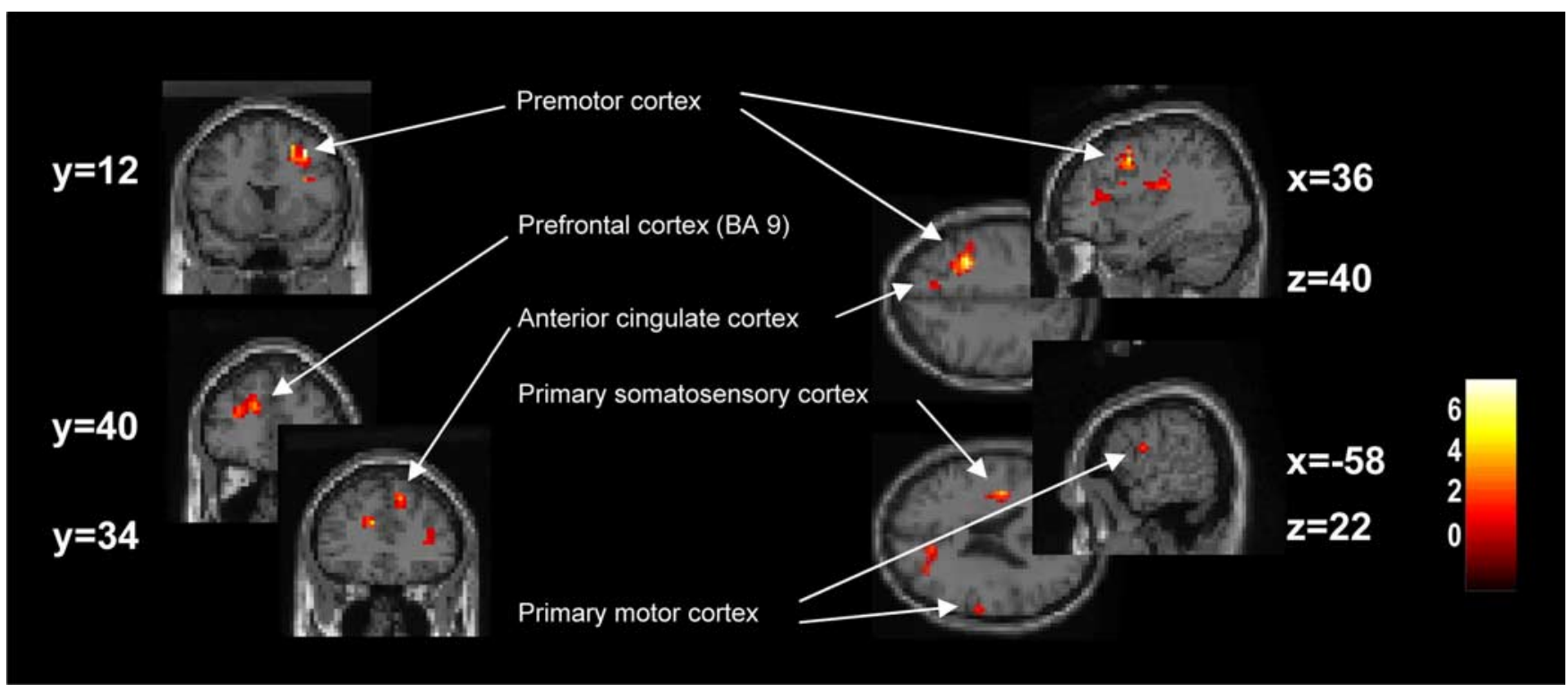

Figure 3. Brain regions showing significant sleep-related decreases in BOLD contrast during retrieval testing of the finger-tapping task with reference to initial training before a $48 \mathrm{~h}$ retention interval $(n=8)$. Note that, because performance of this task elicits strong activation in the respective centers of the ipsilateral and contralateral primary motor and somatosensory cortices, the decreases in BOLD signal inevitably remain confined to the surrounding areas rather than to the centers of activity. Statistical parametric maps are thresholded at $p<0.005$ (uncorrected) and displayed as horizontal, sagittal, and coronal projections superimposed on a standard MNI brain.

Table 3. Coordinates of local maxima for brain regions in which activation during finger motor performance was larger at retrieval testing compared with learning before a $48 \mathrm{~h}$ retention interval $(n=8)$

\begin{tabular}{|c|c|c|c|}
\hline Brain region & Coordinates of peak activation & $Z$ & $p_{\text {SVC }}$ \\
\hline \multicolumn{4}{|c|}{ Areas in which activation increased more in the posttraining sleep than wake condition } \\
\hline \multicolumn{4}{|c|}{ Parietal cortex } \\
\hline L BA 7 (Superior parietal cortex) & $-24,-46,58$ & 3.71 & 0.048 \\
\hline \multicolumn{4}{|l|}{ Subcortical activations } \\
\hline R caudate nucleus & $16,24,14$ & 3.13 & 0.225 \\
\hline L cerebellum & $-18,-44,-18$ & 3.16 & 0.103 \\
\hline \multicolumn{4}{|c|}{ Areas in which activation increased more in the posttraining wake than sleep condition } \\
\hline \multicolumn{4}{|c|}{ Prefrontal cortex } \\
\hline RBA 10 & $34,52,8$ & 2.88 & 0.179 \\
\hline \multicolumn{4}{|l|}{ Subcortical activations } \\
\hline L Putamen & $-16,4,4$ & 3.05 & 0.138 \\
\hline \multicolumn{4}{|c|}{$\begin{array}{l}\text { Areas in which activation increased in the posttraining sleep and wake condition } \\
\text { Primary motor cortex }\end{array}$} \\
\hline RBA 4 & $36,-22,66$ & 3.01 & 0.024 \\
\hline
\end{tabular}

All results are significant at $p<0.005$, uncorrected. $R$, Right hemisphere; L, left hemisphere; BA, Brodmann area.

generated by converting sequential information held in prefrontal working memory networks (Ohbayashi et al., 2003).

A distinct decrease in brain activation at retrieval on the posttraining sleep condition was observed in the left (ipsilateral) M1. Although this decrease was not confirmed with a statistical threshold corrected for multiple testing and, hence, has to be interpreted cautiously, it appears to be of relevance, because this area is known to be critically involved in the processing of complex finger movements even more so in right-handed subjects performing with the left hand (Kim et al., 1993; Chen et al., 1997). Moreover, our finding of a diminished recruitment of the ipsilateral M1, at first glance, contrasts with findings of an extended motor memory representation within this region in persons, such as professional piano players, who have frequently trained a certain motor skill over a long time (Elbert et al., 1995; Karni et al., 1995; Hund-Georgiadis and von Cramon, 1999). Because, in our experiments, the amount of practice was kept constant on both conditions, the expansion of skill representation over a larger
Table 4. Coordinates of local maxima for brain regions in which activation during finger motor performance decreased from new learning to immediate retesting after an $8 \mathrm{~h}$ retention interval of sleep and nocturnal wakefulness (supplementary experiments; $n=10$ )

\begin{tabular}{lll}
\hline Brain region & Coordinates of peak activation & $Z$ \\
\hline $\begin{array}{l}\text { Areas in which activation decreased more in the sleep than in the wake condition } \\
\text { Supplementary motor cortex }\end{array}$ & \\
$\quad$ R BA 6 & $6,18,68$ & 3.53 \\
R BA 6 & $6,-10,76$ & 2.51 \\
Lateral premotor cortex & & 3.07 \\
R BA 6 & $34,18,36$ & \\
Temporal cortex & & 2.69 \\
R BA 37 & $58,-44,0$ & 2.67 \\
R BA 37 & $52,-58,-14$ & 3.15 \\
L BA 37 & $-58,-62,-8$ & 3.48 \\
L BA 37 & $-52,-40,0$ & \\
Parietal cortex & & 2.58 \\
R BA 7 (precuneus) & $20,-52,50$ & 3.30 \\
Subcortical activations & & 2.85 \\
R globus pallidus & $32,-6,0$ & 3.23 \\
L caudate nucleus & $-24,-32,0$ & 2.85 \\
L cerebellum & $-32,-50,-24$ & \\
L cerebellum & $-22,-62,-48$ &
\end{tabular}

Areas in which activation decreased more in the wake than in the sleep condition

No suprathreshold clusters

Areas in which activation decreased more in the posttraining sleep than wake condition across both the $48 \mathrm{~h}$ and the $8 \mathrm{~h}$ retention interval

Prefrontal cortex
LBA 9
$-14,38,26$

Lateral premotor cortex RBA 6 $34,4,42$ 4.28

All results are significant at $p<0.005$, uncorrected. $R$, Right hemisphere; L, left hemisphere; BA, Brodmann area.

cortical area in those persons can be regarded as a consequence of frequent use of the skill, whereas storing into memory, per se, leads to a shrinking of respective network representations (Maquet, 2001). Alternatively, the decrease in ipsilateral M1 could also reflect that the shaping effect of sleep expresses in a more lateralized (i.e., effector-dependent) representation of the 
learned motor sequence (Hikosaka et al., 1999; Korman et al., 2003). Finally, it should be mentioned that the view that practicing a skill independently of any consolidation leads to an increased recruitment of M1 indeed finds support also from our observation of an increased BOLD contrast in the contralateral hand area at retesting on both the sleep and the wake conditions.

A consistent increase in brain activation at retesting selectively after posttraining sleep was found in the left superior parietal cortex. In light of similar increases in parietal cortical activity found in previous studies in the course of learning (Graybiel, 1998; Sakai et al., 1998; Peigneux et al., 2000; Maquet et al., 2003), this finding could point to a reorganizing influence of sleep-dependent memory consolidation on skill representations such that structures supporting automated performance become increasingly involved. There were sleepdependent changes of activity in some additional brain areas (Tables 2, 3). However, because these changes did not reach a corrected statistical threshold, any specific functional interpretation remains tentative in these cases.

One might argue that the changes in brain activation observed here at retrieval in the posttraining sleep condition are linked to enhanced performance rather than to changes during a sleep-dependent consolidation process. However, this is unlikely in light of a number of previous studies that examined rate effects on different motor tasks and showed consistently an increased rather than decreased cortical activation as a function of increased finger movement frequency mainly in M1, the cerebellum, and additional motor areas including different premotor and cingular areas (Blinkenberg et al., 1996; Jenkins et al., 1997; Deiber et al., 1999; Lutz et al., 2005). To exclude confounding effects of speed during task performance, paced versions of the finger-sequence tapping task have been used (Karni et al., 1995, 1998), which, however, do not permit the concurrent monitoring of performance gains during brain scanning, and add a sensory demand to the task (Deiber et al., 1999). Interestingly, in a most recent study, Walker et al. (2005) examined the effects of posttraining sleep on finger sequence tapping and, at retrieval testing in the MRI scanner, introduced such an auditory pacing to avoid confounding effects of speed. Expectedly, the pattern of brain activity induced by the paced task differed in several aspects from the pattern observed in the present study. Nevertheless, the authors came to conclude similarly, that the decreased need for online monitoring of skill performance after posttraining sleep expresses itself in a reduced activation of prefrontal (in this case, frontopolar) regions. However, findings of that study also differed from the present study in that they show sleep-induced increases in activation of the right (contralateral) M1 and anterior medial prefrontal cortices. Although these divergences might, in fact, be related to the pacing introduced at retrieval testing, it remains generally difficult to predict from these experiments the confounding influence speed may have on measuring the memory effects of sleep on sequence tapping under unconstrained conditions. Of particular relevance in this regard
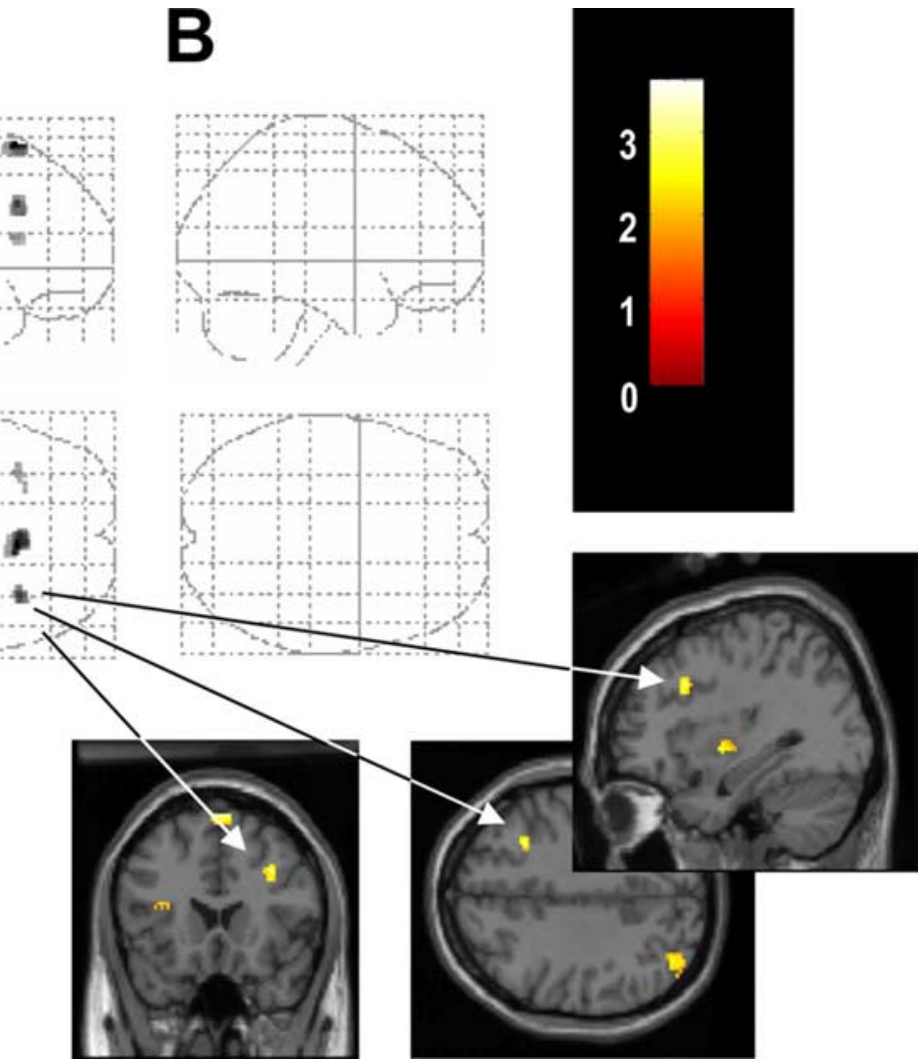

rametric maps (SPMs) of different contrasts obtained in supplementary experiments ( $8 \mathrm{~h}$ retention interval; $n=10)$. Retrieval in these experiments took place immediately after $8 \mathrm{~h}$ periods of sleep and sleep deprivation. $A$, Brain regions in which the decrease in retrieval-related activation with reference to training was larger in the nocturnal sleep than in the nocturnal wake condition and, vice versa, in which this decrease was larger in the nocturnal wake than in the nocturnal sleep condition $(\boldsymbol{B})$ (which never happened). SPMs are thresholded at $p<0.005$ (uncorrected) and displayed as in Figure 3.

is a recent study of finger tapping under unpaced conditions by Lutz et al. (2005). This study confirmed an increased activation in cortical and cerebellar motor regions with increased speed. Interestingly, the rate effects were closely comparable for the dominant and nondominant hand, although the latter was slower, indicating that the processing demand (i.e., the effort to be as fast as possible) rather than the actual physical speed of tapping determines the rate effect. This view agrees well with our results from the comparison of low and high performers on the finger-sequence tapping task at initial training, which revealed comparable activation patterns for both groups and, importantly, no area showing decreased activation with increased tapping rate. Although the issue cannot be entirely resolved, in combination, the available data strongly argue against the view that the reduced activation observed here, in different frontocortical areas at retrieval testing after sleep, reflects increased speed at task performance.

The overall substantial changes in the BOLD signal in cortical and striatal networks indicate that the latent consolidation process during posttraining sleep leads to an essential reorganization of the skill representation. It is plausible to assume that such gross representational changes rely on a coherent but "covert" (not inducing actual movements) reactivation of parts of the representation rather than representing a mere accumulation of plastic changes at isolated synapses (Maquet, 2001; Benington and Frank, 2003; Wagner et al., 2004). Compelling evidence that brain structures activated during learning are reactivated during subsequent REM sleep has been provided in humans using positron emission tomography (Maquet et al., 2000; Peigneux et al., 2003). We thus conclude that sleep- 
dependent consolidation of motor skills represents an active process of storage, shaping the neuronal representation in the absence of practice. Contrasting with practice-dependent changes in cortical skill representation, the process of storing manifests mainly in a more sparse use of frontal cortical tissue for skill representation (Strata et al., 2001). In conjunction with an increased involvement of parietal cortical structures, the sleep-dependent reorganization allows a more efficient and automated behavioral regulation expressed in a substantially improved skill performance after sleep.

\section{References}

Benington JH, Frank MG (2003) Cellular and molecular connections between sleep and synaptic plasticity. Prog Neurobiol 69:71-101.

Blinkenberg M, Bonde C, Holm S, Svarer C, Andersen J, Paulson OB, Law I (1996) Rate dependence of regional cerebral activation during performance of a repetitive motor task: a PET study. J Cereb Blood Flow Metab 16:794-803.

Chen R, Gerloff C, Hallett M, Cohen LG (1997) Involvement of the ipsilateral motor cortex in finger movements of different complexities. Ann Neurol 41:247-254.

Dechent P, Merboldt KD, Frahm J (2004) Is the human primary motor cortex involved in motor imagery? Brain Res Cogn Brain Res 19:138-144.

Deiber MP, Honda M, Ibanez V, Sadato N, Hallett M (1999) Mesial motor areas in self-initiated versus externally triggered movements examined with fMRI: effect of movement type and rate. J Neurophysiol 81:3065-3077.

Drummond SP, Brown GG, Gillin JC, Stricker JL, Wong EC, Buxton RB (2000) Altered brain response to verbal learning following sleep deprivation. Nature 403:655-657.

Elbert T, Pantev C, Wienbruch C, Rockstroh B, Taub E (1995) Increased cortical representation of the fingers of the left hand in string players. Science 270:305-307.

Fischer S, Hallschmid M, Elsner AL, Born J (2002) Sleep forms memory for finger skills. Proc Natl Acad Sci USA 99:11987-11991.

Friston KJ, Penny WD, Glaser DE (2005) Conjunction revisited. NeuroImage 25:661-667.

Gais S, Plihal W, Wagner U, Born J (2000) Early sleep triggers memory for early visual discrimination skills. Nat Neurosci 3:1335-1339.

Gehring WJ, Knight RT (2000) Prefrontal-cingulate interactions in action monitoring. Nat Neurosci 3:516-520.

Grafton ST, Hazeltine E, Ivry RB (2002) Motor sequence learning with the nondominant left hand. A PET functional imaging study. Exp Brain Res 146:369-378.

Graybiel AM (1998) The basal ganglia and chunking of action repertoires. Neurobiol Learn Mem 70:119-136.

Hikosaka O, Nakahara H, Rand MK, Sakai K, Lu X, Nakamura K, Miyachi S, Doya K (1999) Parallel neural networks for learning sequential procedures. Trends Neurosci 22:464-471.

Hoshi E, Tanji J (2000) Integration of target and body-part information in the premotor cortex when planning action. Nature 408:466-470.

Hund-Georgiadis M, von Cramon DY (1999) Motor-learning-related changes in piano players and non-musicians revealed by functional magnetic-resonance signals. Exp Brain Res 125:417-425.

Janke W, Debus G (1978) Die Eigenschaftswörterliste EWL. Göttingen: Hogrefe.

Jenkins IH, Brooks DJ, Nixon PD, Frackowiak RS, Passingham RE (1994) Motor sequence learning: a study with positron emission tomography. J Neurosci 14:3775-3790.

Jenkins IH, Passingham RE, Brooks DJ (1997) The effect of movement frequency on cerebral activation: a positron emission tomography study. J Neurol Sci 151:195-205.

Jueptner M, Stephan KM, Frith CD, Brooks DJ, Frackowiak RS, Passingham RE (1997a) Anatomy of motor learning. I. Frontal cortex and attention to action. J Neurophysiol 77:1313-1324.

Jueptner M, Frith CD, Brooks DJ, Frackowiak RS, Passingham RE (1997b) Anatomy of motor learning. II. Subcortical structures and learning by trial and error. J Neurophysiol 77:1325-1337.

Karni A, Sagi D (1993) The time course of learning a visual skill. Nature 365:250-252.

Karni A, Meyer G, Jezzard P, Adams MM, Turner R, Ungerleider LG (1995) Functional MRI evidence for adult motor cortex plasticity during motor skill learning. Nature 377:155-158.

Karni A, Meyer G, Rey-Hipolito C, Jezzard P, Adams MM, Turner R, Unger- leider LG (1998) The acquisition of skilled motor performance: fast and slow experience-driven changes in primary motor cortex. Proc Natl Acad Sci USA 95:861-868.

Kim SG, Ashe J, Hendrich K, Ellermann JM, Merkle H, Ugurbil K, Georgopoulos AP (1993) Functional magnetic resonance imaging of motor cortex: hemispheric asymmetry and handedness. Science 261:615-617.

Korman M, Raz N, Flash T, Karni A (2003) Multiple shifts in the representation of a motor sequence during the acquisition of skilled performance. Proc Natl Acad Sci USA 100:12492-12497.

Lutz K, Koeneke S, Wustenberg T, Jancke L (2005) Asymmetry of cortical activation during maximum and convenient tapping speed. Neurosci Lett 373:61-66.

Maquet P (2001) The role of sleep in learning and memory. Science 294:1048-1052.

Maquet P, Laureys S, Peigneux P, Fuchs S, Petiau C, Phillips C, Aerts J, Del Fiore G, Degueldre C, Meulemans T, Luxen A, Franck G, Van Der LM, Smith C, Cleeremans A (2000) Experience-dependent changes in cerebral activation during human REM sleep. Nat Neurosci 3:831-836.

Maquet P, Schwartz S, Passingham R, Frith C (2003) Sleep-related consolidation of a visuomotor skill: brain mechanisms as assessed by functional magnetic resonance imaging. J Neurosci 23:1432-1440.

McGaugh JL (2000) Memory: a century of consolidation. Science 287:248251.

Ohbayashi M, Ohki K, Miyashita Y (2003) Conversion of working memory to motor sequence in the monkey premotor cortex. Science 301:233-236.

Oldfield RC (1971) The assessment and analysis of handedness: the Edinburgh inventory. Neuropsychologia 9:97-113.

Peigneux P, Maquet P, Meulemans T, Destrebecqz A, Laureys S, Degueldre C, Delfiore G, Aerts J, Luxen A, Franck G, Van Der Linden M, Cleeremans A (2000) Striatum forever, despite sequence learning variability: a random effect analysis of PET data. Hum Brain Mapp 10:179-194.

Peigneux P, Laureys S, Fuchs S, Destrebecqz A, Collette F, Delbeuck X, Phillips C, Aerts J, Del Fiore G, Degueldre C, Luxen A, Cleeremans A, Maquet P (2003) Learned material content and acquisition level modulate cerebral reactivation during posttraining rapid-eye-movements sleep. NeuroImage 20:125-134.

Penhune VB, Doyon J (2002) Dynamic cortical and subcortical networks in learning and delayed recall of timed motor sequences. J Neurosci 22:1397-1406.

Rechtschaffen A, Kales A (1968) A manual of standardized terminology, techniques and scoring system for sleep stages of human subjects. Los Angeles: Brain Information Service.

Sakai K, Hikosaka O, Miyauchi S, Takino R, Sasaki Y, Putz B (1998) Transition of brain activation from frontal to parietal areas in visuomotor sequence learning. J Neurosci 18:1827-1840.

Seitz RJ, Roland E, Bohm C, Greitz T, Stone-Elander S (1990) Motor learning in man: a positron emission tomographic study. NeuroReport 1:57-60.

Shadmehr R, Holcomb HH (1997) Neural correlates of motor memory consolidation. Science 277:821-825.

Stickgold R, James L, Hobson JA (2000) Visual discrimination learning requires sleep after training. Nat Neurosci 3:1237-1238.

Strata F, Blake DT, Merzenich MM (2001) Sleep restores the response specificity of physiological sensory maps. Soc Neurosci Abstr 27:396.7.

Toni I, Krams M, Turner R, Passingham RE (1998) The time course of changes during motor sequence learning: a whole-brain fMRI study. NeuroImage 8:50-61.

Ungerleider LG, Doyon J, Karni A (2002) Imaging brain plasticity during motor skill learning. Neurobiol Learn Mem 78:553-564.

Wagner U, Gais S, Haider H, Verleger R, Born J (2004) Sleep inspires insight. Nature 427:352-355.

Walker MP (2004) A refined model of sleep and the time course of memory formation. Behav Brain Sci 28:51-64.

Walker MP, Brakefield T, Morgan A, Hobson JA, Stickgold R (2002) Practice with sleep makes perfect: sleep-dependent motor skill learning. Neuron 35:205-211.

Walker MP, Brakefield T, Hobson JA, Stickgold R (2003) Dissociable stages of human memory consolidation and reconsolidation. Nature 425:616-620.

Walker MP, Stickgold R, Alsop D, Gaab N, Schlaug G (2005) Sleepdependent motor memory plasticity in the human brain. Neuroscience 133:911-917.

Wolpert DM, Ghahramani Z, Jordan MI (1995) An internal model for sensorimotor integration. Science 269:1880-1882. 